\section{Lateral canthotomy orbitotomy: a rapid approach to the orbit}

S Hamed-Azzam, DH Verity and GE Rose

Abstract

Purpose The lateral compartment of the orbit can readily be accessed through a horizontal lateral canthotomy without the need to swing the lid or remove bone. In this paper the technique, accessible orbital territory, and duration of surgery are presented.

Patients and methods Retrospective, noninterventional descriptive case series for patients who underwent a lateral canthotomy to access pathology within the lateral orbit. Results A series of 18 patients are included, all presenting with pathology lateral to, or within, the optic nerve. Pathologies included amyloidosis (1), lymphoma (4), metastatic adenocarcinoma within the optic nerve (1), idiopathic lateral rectus muscle mysositis (4), meningothelial meningioma of the optic nerve (1), intraconal orbital meningioma (1), reactive lymphoid hyperplasia (1), optic nerve glioma (3), optic nerve meningioma (1), and cavernous haemangioma (1). The median surgical time was $36 \mathrm{~min}$ (range $23-75 \mathrm{~min}$ ). No patient required detachment of the lower lid, the technique leaving both upper and lower 'arms' of the lateral canthal tendon attached to Whitnall's tubercle.

Conclusions The lateral canthotomy approach orbitotomy is a rapid, safe, and minimally disruptive approach for accessing pathology in the lateral orbit and optic nerve. The lateral canthal tendon is split along the horizontal raphe without detachment of either limb from Whitnall's tubercle, no bone is removed, and the post-operative recovery is rapid with minimal associated inflammation or chemosis. This approach is also flexible, permitting the clinician to increase exposure to the orbit peroperatively by swinging the lower lid if required.

Eye (2018) 32, 333-337; doi:10.1038/eye.2017.173; published online 1 September 2017

\section{Introduction}

There are six main approaches to orbitotomy, all through incisions concealed within periorbital rhytids or conjunctiva, and they allow wide access to most parts of the orbit.

Kronlein's early description of the lateral orbitotomy was a major advance in the management of orbital disease, ${ }^{1}$ but the long, crescent-shaped incision resulted in unsightly scarring, and many other approaches have since been proposed. ${ }^{2-5}$

The lateral parts of the orbit can, however, be readily reached through a horizontal lateral canthotomy without the need to mobilize bone or divide the crura of the lateral canthal tendons. This canthotomy approach gives access lateral to the optic nerve, both the intra- and extra-conal space, and allows tissue sampling from several diverse tissues where there is diffuse intraorbital pathology.

This technique, and the location of pathology which can be accessed, is illustrated with a series of patients undergoing such surgery at Moorfields Eye Hospital.

\section{Materials and methods}

Retrospective, non-interventional descriptive case series of patients who underwent lateral canthotomy orbitotomy, with review of the medical records, pathology, surgical timing, and recovery period. All surgeries were performed by two experienced orbital surgeons (DHV, GER).

\section{Surgical technique}

Scissors are used to divide the outer canthus $1-1.5 \mathrm{~cm}$ horizontally (to the bone) and $2 / 0$ silk traction sutures placed through the upper and lower canthal tissues, thereby creating a rhomboid entry site (Figure 1a); neither the upper nor lower canthi are detached from the orbital rim (Figure 1b). Using two paddle retractors, and with diathermy and blunt dissection through the fat, pathology within the lateral extra-conal and intraconal spaces may be accessed. Canthotomy closure is quickly achieved by lid margin alignment, apposition of
Orbital Service, Moorfields Eye Hospital, NHS Foundation Trust, London, UK

Correspondence: DH Verity, Orbital Service, Moorfields Eye Hospital, NHS Foundation Trust, City Road, London EC1V 2PD, UK

Tel: +44 2072533411 ;

Fax: +44 2076812126.

E-mail: david.verity@

moorfields.nhs.uk

Received: 18 December 2016

Accepted in revised form:

2 July 2017

Published online:

1 September 2017 

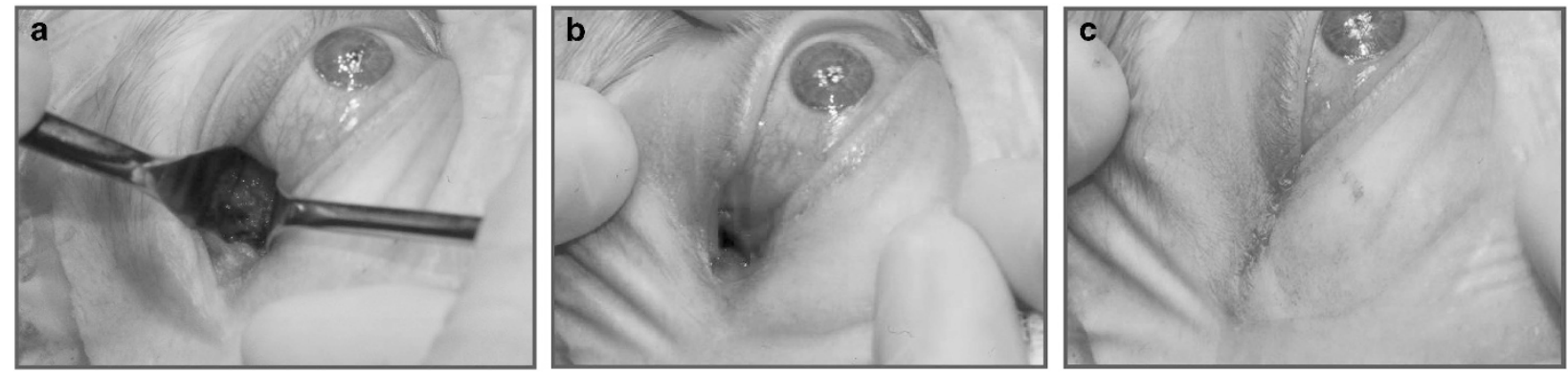

Figure 1 (a) Lateral canthotomy incision creates a rhomboid entry site. (b) No division of the upper or lower lid attachments to the orbital rim. (c) Rapidly achieved closure of the canthotomy.
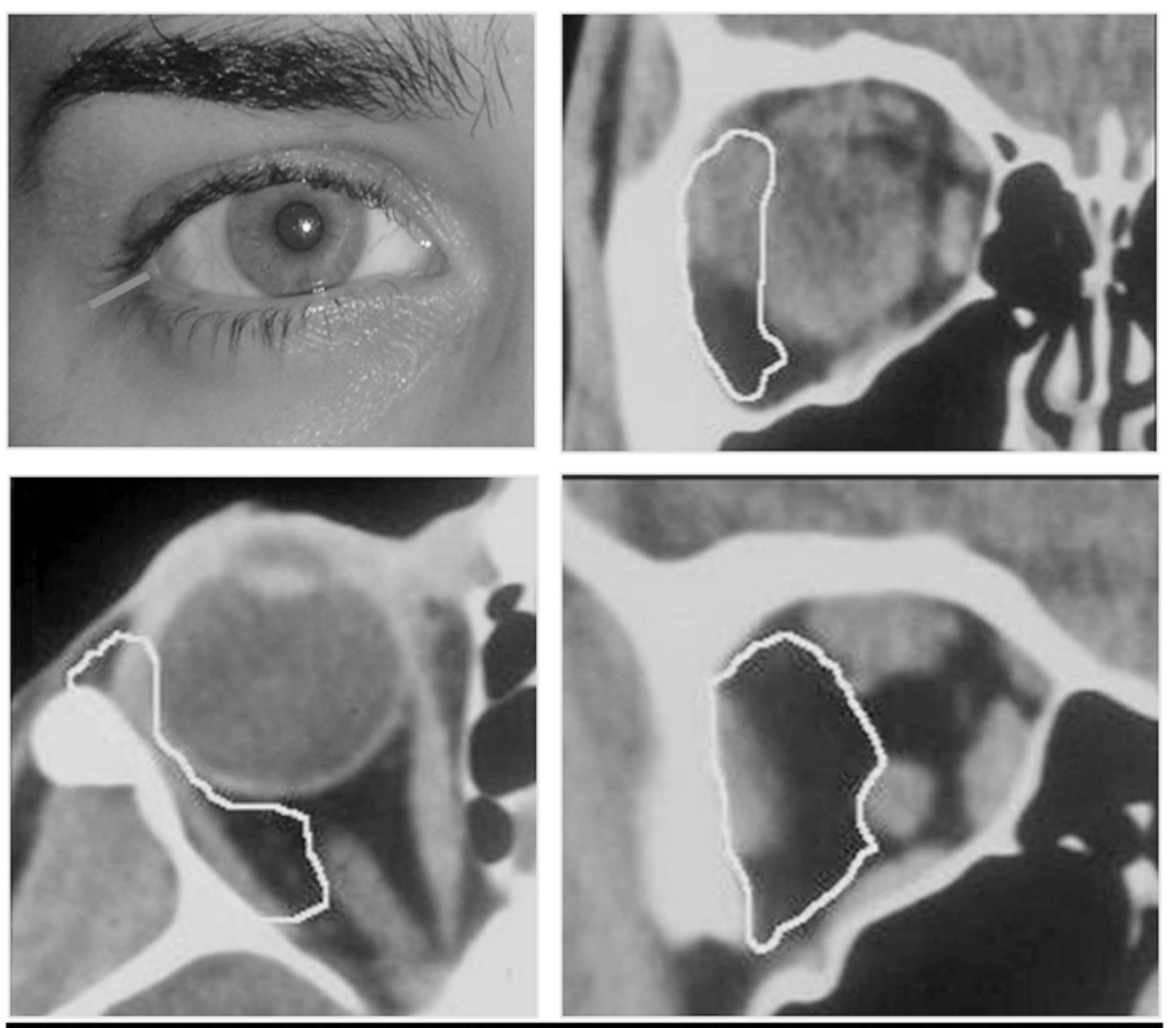

Figure 2 The lateral canthotomy approach gives useful access to the lateral half of the orbit.

the upper and lower limbs of the tendon, and skin closure -all using a single 6-0 soluble suture (Figure 1c).

The technique gives an access for biopsy or debulking of pathology within the lateral two-thirds of the orbit; where greater access is required, the lower canthal tendon can be divided to convert it to a 'low' lower lid swinging flap (Figure 2). ${ }^{6}$

\section{Results}

Medical records and imaging were available for 18 patients having orbitotomy through a lateral canthotomy.
Nine patients had lesions in the intraconal space, 5 in the lateral rectus, 4 had complete excision of optic nerve glioma/meningioma, and 5 had pathology biopsied from several tissues (Table 1; Figure 3). Most lesions were neoplastic (five malignant, eight benign), four had inflammation and one had localized amyloid infiltration.

\section{Surgical time}

The intraoperative time ranged from 23 to $75 \mathrm{~min}$ for biopsies (mean 42; median $36 \mathrm{~min}$ ) and $66 \mathrm{~min}$ average for excision of the orbital optic nerve. There were no 
Table 1 Type of pathology and location in 18 patients undergoing lateral canthotomy orbitotomy

\begin{tabular}{lll}
\hline Location & Pathology & Number of cases \\
\hline Posterior surface of the globe, adjacent to optic nerve & Amyloidosis & 1 \\
Adjacent to the optic nerve & Cavernous haemangioma & 1 \\
Optic nerve & Metastatic adenocarcinoma & 1 \\
Optic nerve & Meningothelial meningioma & 1 \\
Posterior third intraconal space & Florid reactive lymphoid hyperplasia & 1 \\
Choroidal lesion with retrobulbar extension & Lymphoma & 1 \\
Intraconal and into orbital apex & Orbital meningioma & 1 \\
Intraconally, medial to the lateral rectus & Lymphoma & 2 \\
Lateral rectus & Lymphoma & 1 \\
Lateral rectus & Chronic inflammation & 4 \\
Optic nerve & Complete excision of optic nerve glioma & 3 \\
Optic nerve & Complete excision of optic nerve meningioma & 1 \\
\hline
\end{tabular}

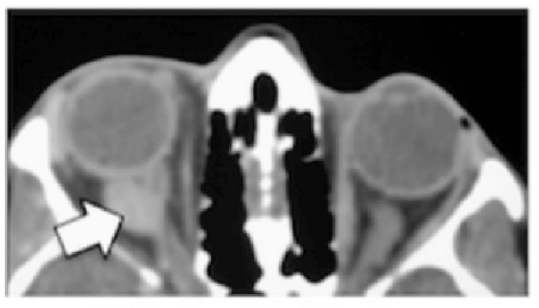

Diffuse large B cell lymphoma

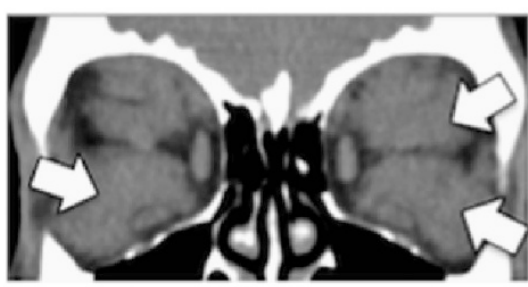

Chronic idiopathic inflammation

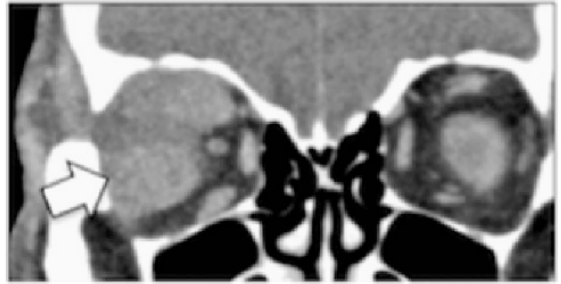

Diffuse large B cell lymphoma

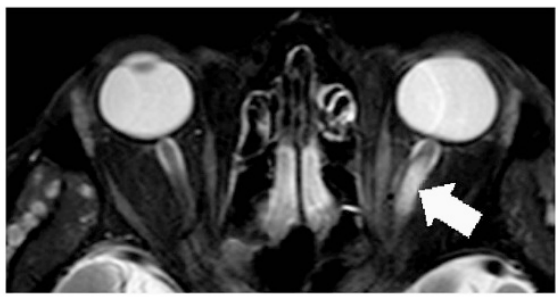

Metastatic Adenocarcinoma

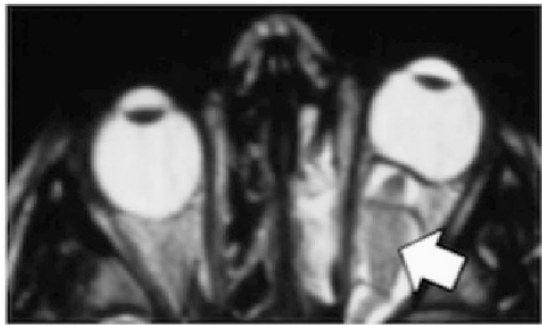

Infantile glioma

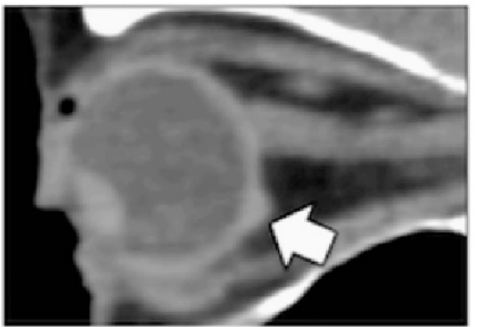

Diffuse large B cell lymphoma

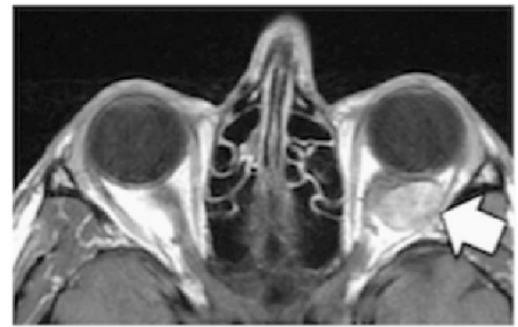

Cavernous haemangioma

Figure 3 Orbital images of some of the included patients with their pathology.

permanent complications attributable to the surgical approach, but with excision of the optic nerve or orbital apex surgery, it was usual to see a global reduction in eye movements for a few weeks after surgery. None of the cases were complicated by hemorrhage, or by unexpected visual loss, mydriasis, diplopia, or abduction deficit.

There were no cases of canthal dystopia and in all cases the incision healed rapidly in the lateral rhytid

(Figure 4).

\section{Discussion}

Historically, bone-swinging lateral orbitotomy was the favored access to the lateral intraconal space and the Kronlein incision (from 1888) was crescent-shaped, with the anterior convexity at the lateral orbital rim, and extending over the temporal fossa toward the ear. ${ }^{1}$ The Kronlein incision leaves a long and visible scar

(Figure 5a), and the approach has been modified over the 


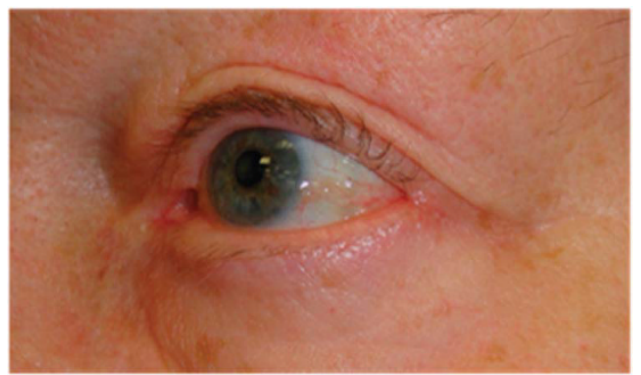

Figure 4 Healing lateral canthotomy incision at 3 months after biopsy of intraconral lesion. Histopathology was consistent with extranodal marginal zone lymphoma.

years. $^{2-8}$ The Kocher approach placed the incision below the brow and turning to the midpoint of the zygomatic $\operatorname{arch}^{7}$ (Figure 5b), and the S-shaped Stallard-Wright incision was partially hidden within the brow (Figure 5c). ${ }^{3,8}$ A modified Kronlein approach for boneswinging lateral orbitotomy has been described using either a horizontal incision through lateral canthus, ${ }^{2,4-5}$ an incision placed laterally in the upper lid skin crease, $^{9-11}$ or a lateral canthal skin incision requiring dedicated micro-instrumentation and an operating microscope. ${ }^{12}$

All the above techniques tend to cause long and visible scars, with various authors subsequently advocating different approaches to minimize scarring, which includes approaches through the upper lid skin crease approach, ${ }^{10,11}$ an incision within the hairline anterior to the ear, ${ }^{13}$ and a coronal scalp flap approach. ${ }^{14}$ Although the coronal approach avoids a visible incision, it carries a risk of injury to the upper divisions of the facial nerve (with entrance to the fat pad superficial to the temporalis muscle in Yasargil's plane), is time-consuming, and generally unnecessary given the alternatives. ${ }^{15}$ Of interest, Nemet et al have proposed the 'lateral triangle flap approach' lateral orbitotomy, this being a combination of a lateral upper lid skin crease incision and a horizontal skin incision lateral to the lateral canthus; such an approach allows access to the lateral and superolateral orbital compartments, with the option of proceeding to a bone-swinging orbitotomy if required. ${ }^{16}$

In contrast, for biopsy or debulking of pathology in the lateral canthal compartment where a biopsy or debulk is required, or for excision of a relatively small lesion, the lateral canthotomy orbitotomy as described here is rapid, safe, and minimally disruptive, giving wide access to the lateral orbit including the retrobulbar space and optic nerve. It is a viable method for optic nerve fenestration, given the relatively posterior entry point to the orbit, compared to the upper lid skin crease and retrocaruncular approaches, the optic nerve is visualized with a greater 'conoid of view' where the access path being shorter. There are many indications for this approach (Table 2),
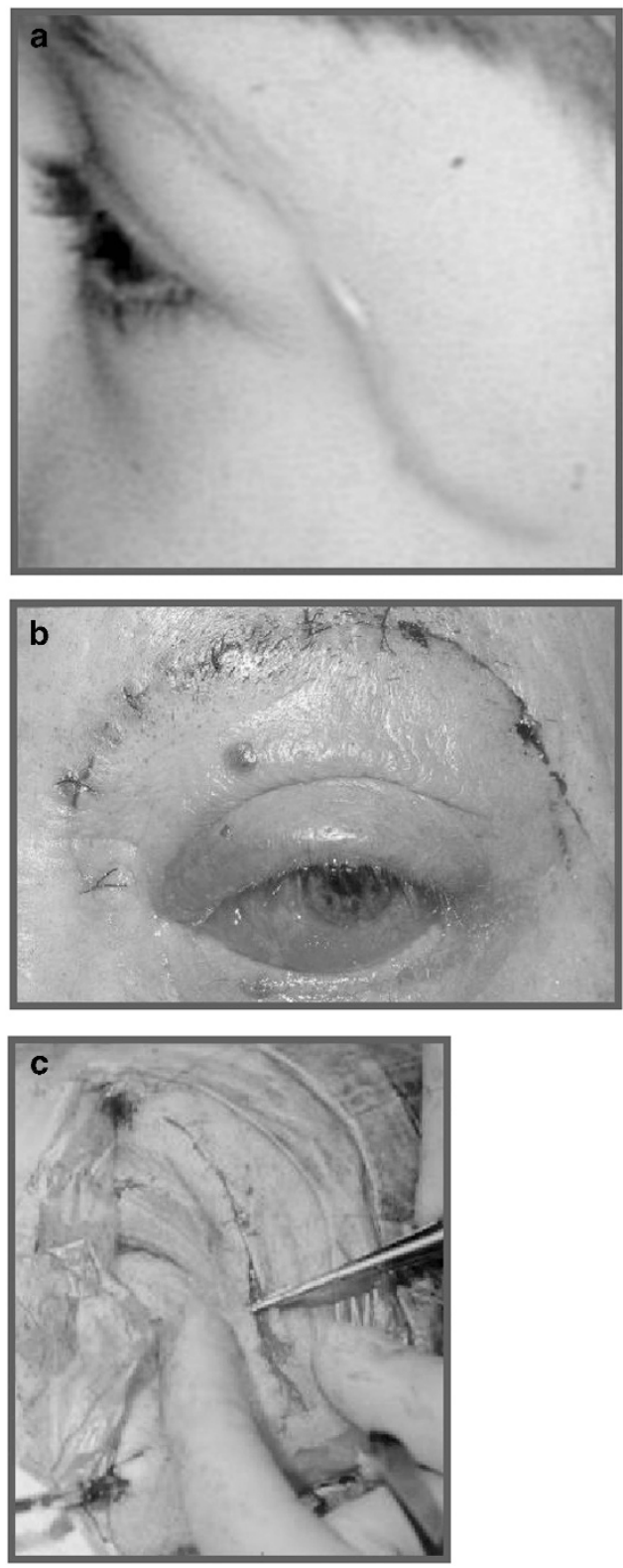

Figure 5 (a) A child was referred with a misplaced Kronlein incision that had resulted in a long and visible scar. (b) Modified Kocher sub-brow incision. (c) Stallard-Wright incision for lateral orbitotomy, in lateral brow hairs and running out across zygomatic arch.

and it has several advantages (Table 3); Chief among its advantages is that-by dividing the tendon along the lateral horizontal raphe without detaching of either limb from Whitnall's tubercle-the surgical time is typically short and post-operative recovery rapid, with minimal post-operative inflammation or chemosis, and no chance of canthal dystopia. Finally, should increased exposure be required, this is readily achieved by detaching the lower limb of the tendon and opening the inferior fornix, thereby converting it to a lower lid swinging flap. 
Table 2 Indications for lateral canthotomy orbitotomy

1. Where a 'triple biopsy' is required in cases of diffuse disease: multiple structures can be biopsied, including lacrimal gland, recti muscles (inferior rectus or lateral rectus), and intra/extra-conal fat or pathology.

2. Pathology affecting the inferior or lateral rectus muscle(s) (eg, lymphoma).

3. Pathology along the lateral orbital wall (eg, sphenoid wing meningioma).

4. Lesions at the orbital apex: the advantage of this approach over the bone-swinging lateral orbitotomy is that bone dissemination of malignancy-where present-is avoided.

5. Optic nerve biopsy or fenestration.

6. Removal of small intraconal pathology.

7. Removal of larger masses from the shallower childhood orbit.

8. Drainage of orbital abscesses along the lateral wall.

Table 3 Advantages of the lateral canthotomy orbitotomy, as compared to other approaches to the lateral part of the orbit

1. Rapid and low risk.

2. Minimal disruption of the lateral canthus and no canthal dystopia when healed.

3. Wide exposure of the lateral compartment of orbit.

4. Almost no post-operative swelling due to the horizontal nature of the incision.

5. Option of converting the approach to a lower lid-swing if increased exposure is required.

6. Option of converting to a bone-swinging orbitotomy.

7. Avoidance of intraosseous dissemination in cases of malignancy.

\section{Summary}

\section{What was known before}

- Classic lateral orbitotomy included orbital bone removal and/or lower lid swinging approach, which prolongs the surgical time, slows post-operative recovery, and can cause lateral cantonal dystopia.

What this study adds

- The surgical technique that is proposed in this paper is rapid, safe, and minimally disruptive, which gives wide access to the lateral orbital compartment.

\section{Conflict of interest}

The authors declare no conflict of interest.

\section{References}

1 Kronlein RU. Zur pathologie und operative behandlung der dermoidcysten der orbita. Bruns Beitr Klin Chir 1888; 4: 149-163.

2 Berke RN. Modified Kronlein operation. AMA Arch Ophthalmol 1954; 51: 609-632.

3 Stallard HB. Surgery of the orbit. Ann R Coll Surg Engl 1968; 43: 125-140.

4 Reese AB. Orbital tumors and their surgical treatment. Am J Ophthalmol 1941; 24: 386-394 497-502.

5 Smith JL. Anterolateral approach to the orbit. Trans Am Acad Ophthalmol Otolaryngol 1971; 75: 1059-1064.
6 Verity DH, Rose GE. Surgical approaches to the orbit. In: Perry JD, Singh AD (eds). Clinical Ophthalmic Pathology. Springer: Heidelberg, 2014.

7 Rootman J, Stewart B, Golbert RA (eds). Orbital Surgerya Conceptual Approach. Lippincott: Philadelphia, 1995.

8 Wright JE. Surgical exploration of the orbit. In: Stewart WB (ed). Ophthalmic Plastic and Reconstructive Surgery. American Academy of Ophthalmology: San Francisco, 1984, pp 309-315.

9 Henderson JW. Orbital Tumors. Raven Press: New York, 1994, pp 425-434.

10 Harris GJ, Logani SC. Eyelid crease incision for lateral orbitotomy. Ophthalmic Plast Reconstr Surg 1999; 15: 9-18.

11 Unal M, Ileri F, Konuk O, Hasanreisogglu B. Balanced orbital decompression in Graves' orbitopathy: upper eyelid crease incision for extended lateral wall decompression. Orbit 2000; 19: 109-117.

12 Maroom JC, Kennerdell JS. Lateral microsurgical approach to intraorbital tumors. J Neurosurg 1976; 44: 556-561.

13 Guyton JS. Decompression of the orbit. Surgery 1946; 19: 790-809.

14 Stewart WB, Levin PS, Toth BA. Orbital surgery. The technique of coronal scalp flap approach to the lateral orbitotomy. Arch Ophthalmol 1988; 106: 1724-1726.

15 Rootman J, Stewart B, Goldberg RA. Regional approach to the anterior, mid and apical orbit. In: Rootman J, Stewart B, Goldberg RA (eds). Orbital Surgery. Lippincott-Raven Publishers: Philadelphia, 1995, pp 228.

16 Nemet A, Martin P. The lateral triangle flap-a new approach for lateral orbitotomy. Orbit 2007; 26: 89-95. 